\title{
Crossing the Blood-Brain-Barrier with Transferrin Conjugated Carbon Dots: A Zebrafish Model Study
}

Shanghao Li ${ }^{1}$, Zhili Peng ${ }^{1}$, Julia Dallman ${ }^{2}$, James Baker $^{2}$, Abdelhameed M. Othman $^{3,4}$, Patrica L. Blackwelder $^{5,6}$, and Roger M. Leblanc ${ }^{1, *}$

${ }^{1}$ Department of Chemistry, University of Miami, 1301 Memorial Drive, Coral Gables, Florida, 33146, United States

${ }^{2}$ Department of Biology, University of Miami, 1301 Memorial Drive, Coral Gables, Florida, 33146, United States

${ }^{3}$ Department of Chemistry, Faculty of Science in Yanbu, Taibah University, Yanbu, Saudi Arabia

${ }^{4}$ Department of Environmental Biotechnology, Genetic Engineering and Biotechnology, University of Sadat City, Sadat City, Egypt

${ }^{5}$ University of Miami Center for Advanced Microscopy and Marine Geosciences, 1301 Memorial Drive, University of Miami, Coral Gables, FL, 33146, United States

${ }^{6}$ Nova Southeastern University Oceanographic Center, 8000 North Ocean Drive, Dania, FL, 33004, United States

*Corresponding author (R.M.L). Tel.: +1-305-284-2194; Fax: + 1-305-284-6367. E-mail: rml@miami.edu. 


\begin{abstract}
Drug delivery to the central nervous system (CNS) in biological systems remains a major medical challenge due to the tight junctions between endothelial cells known as the blood-brain-barrier (BBB). Here we use a zebrafish model to explore the possibility of using transferrin-conjugated carbon dots (C-Dots) to ferry compounds across the BBB. C-Dots have previously been reported to inhibit protein fibrillation, and they are also used to deliver drugs for disease treatment. In terms of the potential medical application of C-Dots for the treatment of CNS diseases, one of the most formidable challenges is how to deliver them inside the CNS. To achieve this in this study, human transferrin was covalently conjugated to C-Dots. The conjugates were then injected into the vasculature of zebrafish to examine the possibility of crossing the BBB in vivo via transferrin receptor-mediated endocytosis. The experimental observations suggest that the transferrin-C-Dots can enter the CNS while C-Dots alone cannot.
\end{abstract}

\title{
Graphical abstract
}

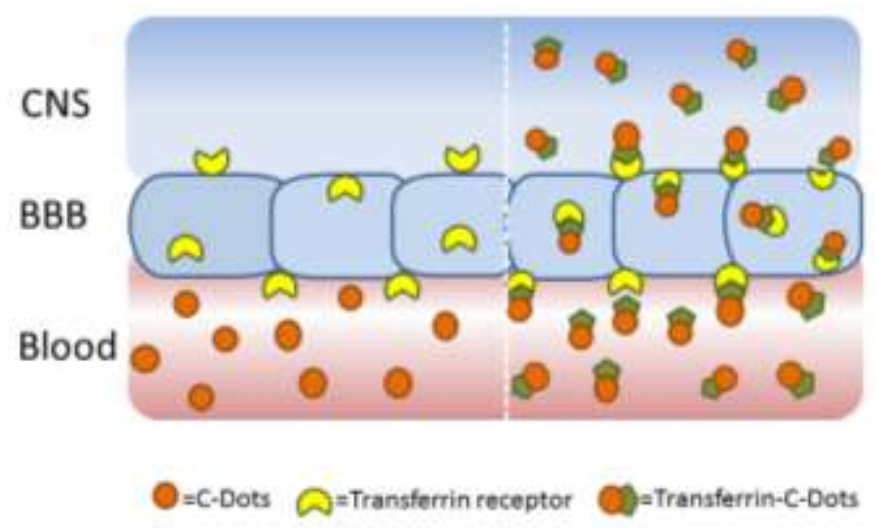

Keywords: blood-brain-barrier; carbon dots; central nervous system; transferrin; 
The central nervous system (CNS), consisting of the brain and spinal cord, is responsible for integrating sensory information and responding accordingly. It has been well recognized that the CNS is protected by the complex and highly regulated blood-brain-barrier (BBB) which serves as a physiological checkpoint to allow the entry of selected molecules from the blood circulation into the CNS. ${ }^{1}$ The BBB is primarily composed of capillary endothelial cells, which are closely interconnected by tight intercellular junctions. ${ }^{2}$ While the capacity of the BBB to restrict permeability and transport into the CNS is protective, it also represents a formidable obstacle for the delivery of therapeutic molecules from the blood to the CNS. Studies show that less than $2 \%$ of small-molecule drugs can cross the BBB, and these drugs usually possess dual characteristics, i.e. molecular weight less than $500 \mathrm{~g} / \mathrm{mol}$ and high lipophilicity. ${ }^{3,4}$ Practically $100 \%$ of largemolecule drugs or nanoparticles targeted for the CNS diseases do not readily cross the BBB unless the surfaces of these drugs or nanoparticles are specifically modified for the uptake. ${ }^{5}$ Because of the inability to adequately deliver therapeutic agents across the BBB, the current treatments for the CNS diseases remain extremely limited. ${ }^{6}$

Compared with invasive drug delivery, non-invasive drug delivery to the CNS achieves better efficacy with lower dosage and side effects by taking advantage of the receptor-mediated transcytosis across the BBB. ${ }^{7}$ Over the last few years, the development of a drug delivery system for crossing the BBB using nanoparticle carriers is among the most notable advances in the research of pharmaceutical research. ${ }^{8,9}$ These carriers are usually conjugated to surface ligands which can be specifically bound by their receptors in the brain capillary endothelial cells. ${ }^{10}$ Transferrin is one such ligand because the presence of highly expressed transferrin receptors on the endothelial cells have been shown to ferry transferrin-conjugates to the CNS. ${ }^{11,12}$ The specific affinity between transferrin and the transferrin receptor as a mechanism to deliver therapeutics to the brain is an area of active exploration, although a precise understanding at the cellular and molecular level remains elusive. ${ }^{13,14}$ It has been reported that transferrin-containing gold nanoparticles can reach the brain parenchyma when administrated systemically. ${ }^{11}$ Most recently, an in vitro study using human brain cancer lines (LN229 and U87) shows that transferrin receptor-targeted gold nanoparticles could deliver the pro-drug into the mitochondria of the glioma cells. ${ }^{15}$ In another study, Jiang et al. demonstrated that in addition to delivering compounds across the $\mathrm{BBB}$, transferrin-conjugated superparamagnetic iron oxide nanoparticles could be internalized by C6 glioma cells and detected by magnetic resonance imaging (MRI). ${ }^{16}$ The delivery of nanoparticles with therapeutic effects in a cell or tissue specific manner may provide better efficacy and lower toxicity for disease treatment. ${ }^{17}$ 
Carbon dots (C-Dots), a new type of nanoparticle with diameters below $10 \mathrm{~nm}$, have recently emerged and attracted extensive attention for their unique optical properties and promising applications in drug delivery. ${ }^{18-20} \mathrm{Li}$ et al. observed in vitro the internalization of the transferrin conjugated C-Dots in the cancer cells HeLa. ${ }^{21}$ More recently, we found that nontoxic C-Dots potently inhibit insulin fibrillation in aqueous solution. ${ }^{22}$ In addition, our current research has also found that these C-Dots suppress fibrillation of amyloid beta peptides. It has been shown previously that proteins and peptides may share a common molecular mechanism to develop fibrils, a process associated with pathologies in CNS diseases including Alzheimer's and Parkinson's diseases. ${ }^{23,24}$ However, to utilize the inhibiting effect of C-Dots on protein fibrillation, they need to be delivered first into the CNS by crossing the BBB. However, only one article up to now has been published on the topic that $\mathrm{C}$-Dots can penetrate the $\mathrm{BBB}$, targeting brain cancer glioma tissue..$^{25}$ The specialty of C-Dots in that publication may originate from the reagents of Dglucose and L-aspartic acid, both of which could be transported to cross the BBB by transporterand/or receptor-mediated uptake. ${ }^{25}$ It is unknown whether C-Dots with different surface properties can cross the BBB.

To address the challenge of crossing the $\mathrm{BBB}$, we conjugated human transferrin to C-Dots to facilitate the delivery of C-Dots across the BBB from the circulation to the CNS. Zebrafish (Danio rerio) is a relatively complex vertebrate species with a high degree of physiological and genetic homology to humans. They also possess all major neurotransmitters, hormones, and receptors, including transferrin. ${ }^{26}$ The remarkable anatomical and physiological conservation in the CNS development and function between zebrafish and amniotes (mouse and humans) has been demonstrated. ${ }^{27}$ Therefore, a zebrafish model enables researchers to not only identify genes that might underlie human disease, but also to test and develop novel therapeutic agents in vivo. ${ }^{28}$ Because of their small size and the relatively simple laboratory culture conditions required, it is much more straightforward to maintain zebrafish in what appear to be more natural conditions than possible to simulate in mammals such as mice. ${ }^{29}$ Another advantage of zebrafish model is the transparence of the body, meaning that it is possible to follow the pharmacological treatment using non-invasive imaging techniques. ${ }^{29}$ Particularly, previous studies also demonstrated that maturation of the zebrafish BBB occurred around 3 days post-fertilisation (d.p.f.), and that this BBB shared both structural and functional similarities with that of mammals. ${ }^{30,31}$ Therefore, larval zebrafish at 6 d.p.f. with mature BBB were selected in our experiments as an in vivo model to examine the possibility of transporting C-Dots into the CNS. Transferrin, dye-transferrin or dye of fluorescein were covalently conjugated with C-Dots, and then the conjugates were injected to 
the zebrafish heart to examine the possibility of the receptor-mediated delivery in the CNS. Experimental observations confirmed that the transferrin-conjugated system facilitated entry of the C-Dots into the CNS.

A schematic summary of the experimental processes is illustrated in Scheme 1. The C-Dots used in this study were synthesized and characterized as in our previous studies. ${ }^{22}$ The prepared C-Dots were characterized by UV-vis absorption, fluorescence, infrared spectroscopy (IR), X-ray photoelectron spectroscopy (XPS), and transmission electron microscopy (TEM). The results from these spectroscopic and microscopic characterizations were almost identical to our previous study (Figure SI-1, Supporting Information). ${ }^{22}$ Please refer to the Supporting Information (SI) for the experimental details and the characterizations. The average particle diameter of C-Dots is approximately $5 \mathrm{~nm}$ with abundant carboxylic groups distributed on the surface for possible chemical modifications with biomolecules. ${ }^{32}$

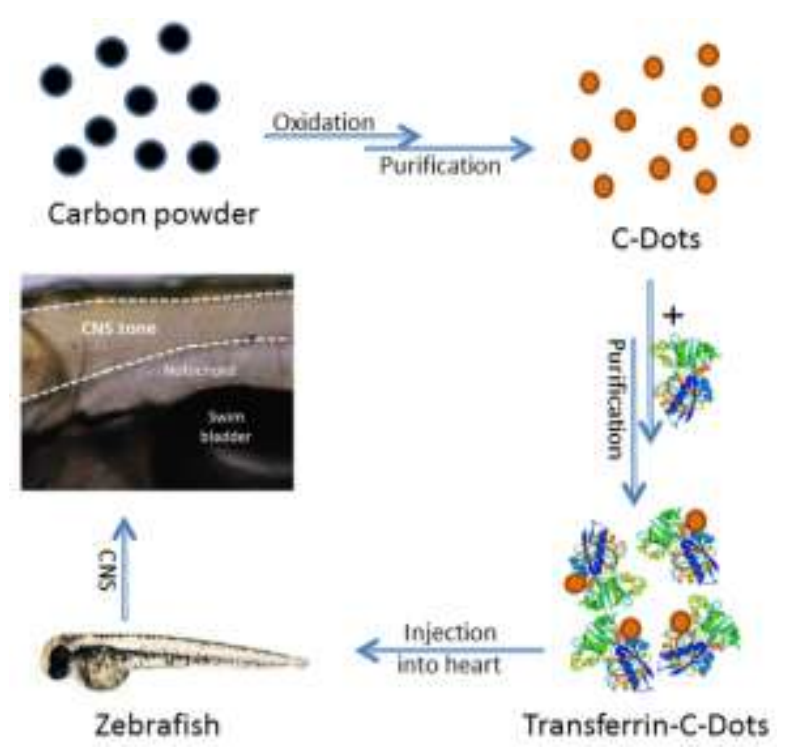

Scheme 1. The experimental processes of carbon dots preparation from carbon powder, transferrin-C-Dots conjugation via EDC/NHS reaction, injection of conjugates to the zebrafish heart, and the confocal fluorescence microscopy imaging of the CNS zone.

In this study, transferrin, dye-labeled transferrin, or fluorescein were first covalently conjugated to $\mathrm{C}$-Dots and tested for their ability to cross the BBB (Scheme 1). After the conjugating reaction, the conjugates were purified by a size exclusion chromatography column. The purified conjugates were systematically characterized by UV-vis absorption, fluorescence, circular dichroism (CD), matrix-assisted laser desorption ionization-time of flight (MALDI-TOF) mass spectroscopy, and 
transmission electron microscopy (TEM). UV-vis absorption spectral differences shown in Figure 1A indicate the successful conjugation of the transferrin-C-Dots and the dye-transferrin-C-Dots. The transferrin-C-Dots have absorption around $260 \mathrm{~nm}$, while the dye-transferrin-C-Dots show a characteristic absorption peak at $594 \mathrm{~nm}$ of the fluorescent dye. Figure 1B demonstrates that the fluorescence spectra of the transferrin-C-Dots display both the fluorescence of the protein transferrin (345 nm, black arrow) and C-Dots (excitation wavelength dependence, red arrows). The emission peak of transferrin at $345 \mathrm{~nm}$ is due to the presence of tryptophan residues when excited at $290 \mathrm{~nm}^{33}$ The excitation wavelength dependent photoluminescence is the key fluorescent characteristic of C-Dots. ${ }^{22,34-36}$ The emission peak of C-Dots was shifted from around 515 to $595 \mathrm{~nm}$ when the excitation was shifted from 290 to $560 \mathrm{~nm}$ (Figure 1B). Similarly, besides the strong fluorescence of the dye at $613 \mathrm{~nm}$, the fluorescence spectra of the dyetransferrin-C-Dots also showed the characteristic shifting emission of C-Dots (red arrows) and transferrin (black arrow, Figure 1C). It is worth noting that the emission of C-Dots at longer wavelength was overlapped by the much stronger fluorescence of the dye at $613 \mathrm{~nm}$. That explains why the emission peak of C-Dots is not shown in Figure 1C when excited at 520 and 560 nm. MALDI-TOF spectra also support the success of conjugation, as the mass determined were 79,224 Da of transferrin, 81,596 Da of dye-transferrin, 83,330 Da of transferrin-C-Dots, and 85,702 Da of dye-transferrin-C-Dots (Figure 1D). All the evidence from the above spectroscopic studies demonstrates the success of covalent conjugation between C-Dots and transferrin. TEM images display that the diameter of bare C-Dots is approximately $5 \mathrm{~nm}$ (Figure 1E). After conjugation with dye-transferrin, the size of the conjugates increases to 10-14 nm (Figure 1F).
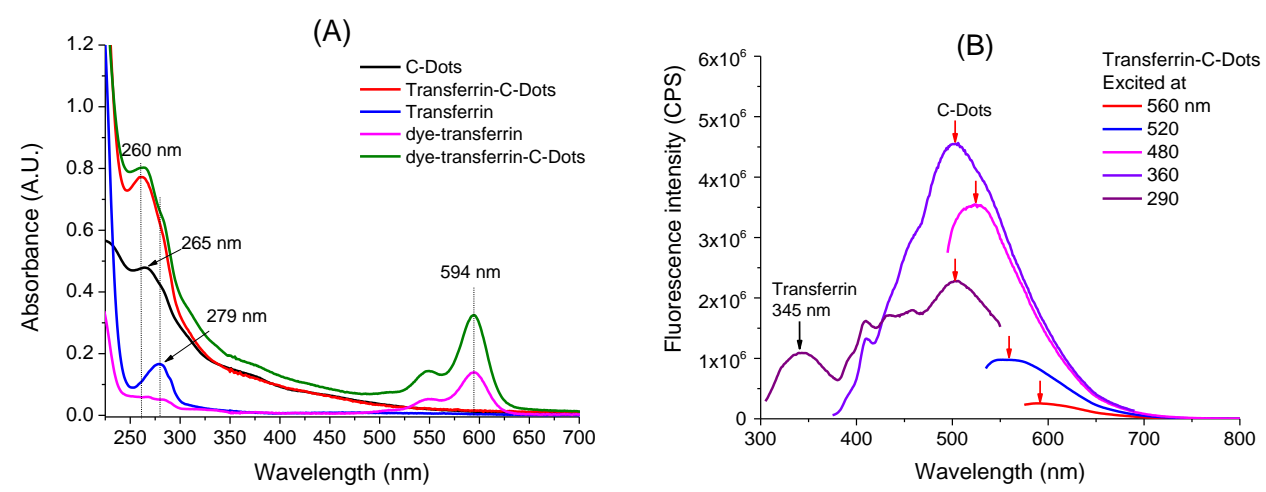

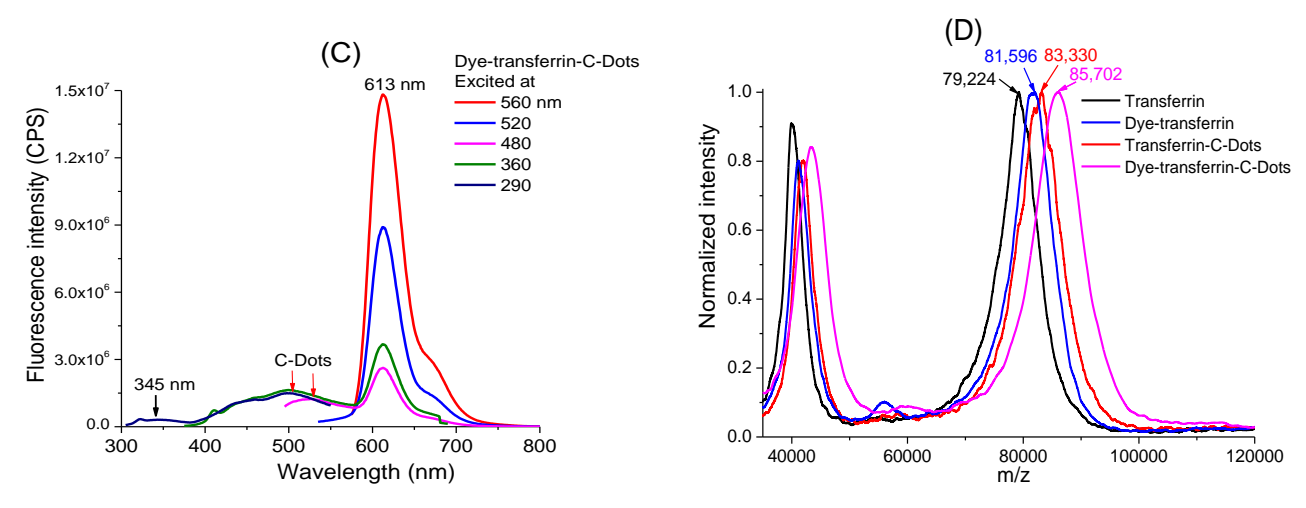

$(\mathrm{E})$
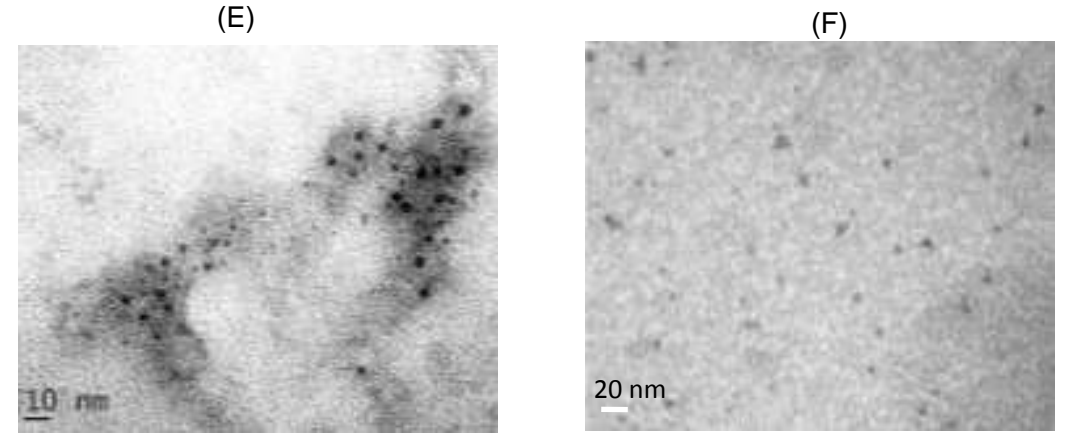

Figure 1. Spectroscopic characterization of transferrin-C-Dots conjugates: (A) UV-vis spectra; (B) Fluorescence spectra of transferrin-C-Dots conjugates; (C) Fluorescence spectra of dyetransferrin-C-Dots conjugates; (D) Normalized MALDI-TOF spectra; (E) TEM image of C-Dots and (F) TEM image of dye-transferrin-C-Dots.

To determine possible conformation and zeta potential changes of transferrin before and after conjugation, the samples were analyzed by circular dichroism spectroscopy and zeta potential. ${ }^{37}$ The normalized spectra do not show appreciable differences among native transferrin, dyetransferrin, transferrin-C-Dots and dye-transferrin-C-Dots (Figure SI-2). Therefore, after the conjugation with C-Dots, the native conformation of transferrin remains in the conjugates. Zeta potential was measured to evaluate the charge differences of transferrin before and after conjugation. The average zeta potential values of C-Dots, transferrin, transferrin-C-Dots, and dye-transferrin-C-Dots in PBS at pH 7.4 are -31.0, -11.9, -17.8, and $-13.4 \mathrm{mV}$, respectively. Since the zeta potential values among transferrin, transferrin-C-Dots and dye-transferrin-C-Dots are similar, the conjugation does not change significantly on the zeta potential. Therefore, the covalent conjugation of transferrin should not affect the binding between transferrin and its receptor in the process of cellular uptake and transcytosis. 
To further test the stability of the transferrin-C-Dots and dye-transferrin-C-Dots under physiological conditions, we measured the conformation of these conjugates in $0.1 \mathrm{M}$ phosphate buffer solution at $\mathrm{pH} 7.4$ using circular dichroism spectroscopy. The data showed that the conformation of transferrin in the conjugates was reserved as the native conformation without notable changes even after 48 hours under room temperature. This observation demonstrated the stability of the conjugates in the buffer in terms of protein conformation.

It is well known that the brain and spinal cord of the central nervous system (CNS) are protected by a unique and complex barrier called blood brain barrier (BBB). This barrier regulates and restricts the free diffusion of molecules or materials to the CNS. ${ }^{1,38}$ The CNS is bathed in cerebral spinal fluid that circulates through ventricles of the brain that are contiguous with the central canal of the spinal cord. ${ }^{39}$ Therefore, once molecules or materials penetrate the BBB to the spinal cord, they can be easily circulated and delivered to the brain. In the light microscopy, the transparent nature of the body of the larval zebrafish and the CNS zone are apparent (Figure 2A and $2 \mathrm{~B})$.

A

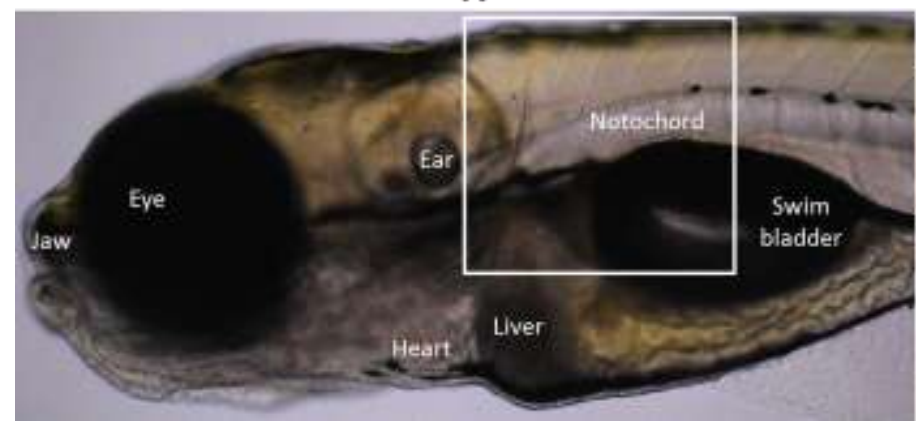

B

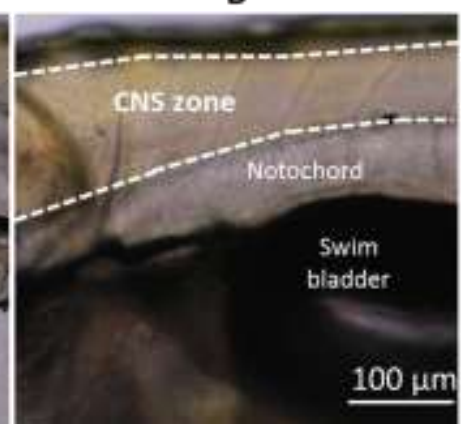

Figure 2. The light micrograph of the zebrafish body at 6 days after fertilization (A); The zone in the box of Figure $2 \mathrm{~A}$ is the CNS zone (B).

C-Dots or the conjugates were injected intravascularly to the heart of the zebrafish to examine their ability to penetrate the CNS. It is worth noting that these C-Dots conjugates including CDots alone have no or very limited cytotoxicity to zebrafish after injection. Experimental observations showed that the fish were still alive and actively swimming 24 hours after injection. As shown in Figure $3 \mathrm{~A} 1$ and A2, the confocal fluorescence images of the control zebrafish without injection reveal that the CNS zone is not fluorescent. Once the C-Dots or the conjugates cross the BBB, they penetrate and enter the CNS. If the fluorescence intensity is strong enough, they would be expected to "light-up" the CNS. However, after C-Dots were injected to the heart 
of zebrafish, no clear difference of fluorescence was observed in the CNS (Figure 3 B2) in comparison to the control experiment (Figure $3 \mathrm{~A} 2$ ). Increasing the amount of the C-Dots injection did not make any difference either. There are two possible reasons for this observation. (1) The fluorescence intensity of C-Dots was too weak to be seen in the CNS. (2) C-Dots did not cross the BBB to enter the CNS. We hence covalently conjugated 5-(aminomethyl) fluorescein to C-Dots (Fluo-C-Dots). After injection of these Fluo-C-Dots to the heart of zebrafish, we observed bright fluorescence in the body (Figure SI-3 D2 in Supporting Information), but no fluorescence was observed in the CNS zone. Collectively considering that essentially $100 \%$ larger molecular weight drug does not cross the $\mathrm{BBB},{ }^{3,4}$ our findings suggest that C-Dots alone as $\sim 5 \mathrm{~nm}$ nanoparticles do not efficiently enter the CNS.
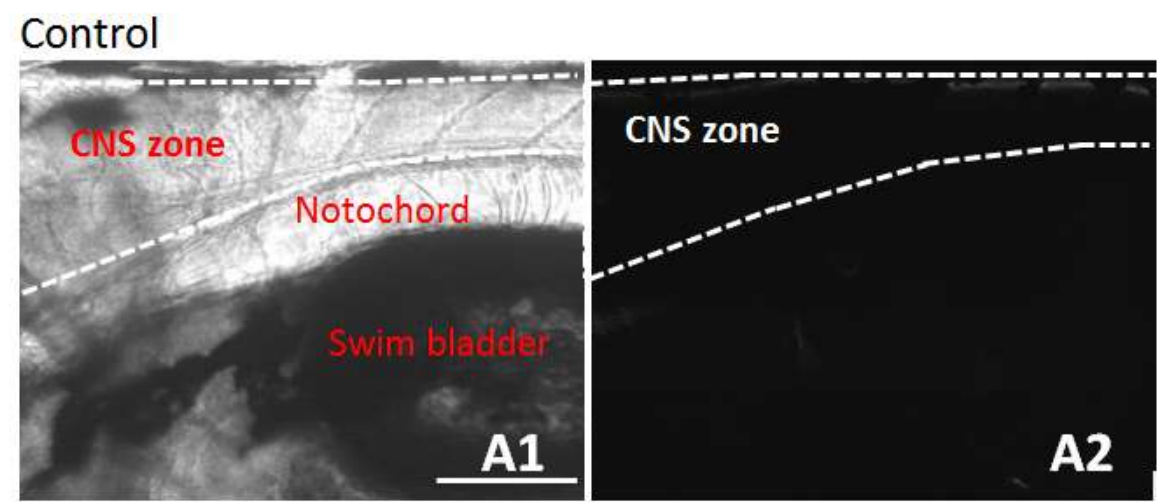

\section{C-Dots}
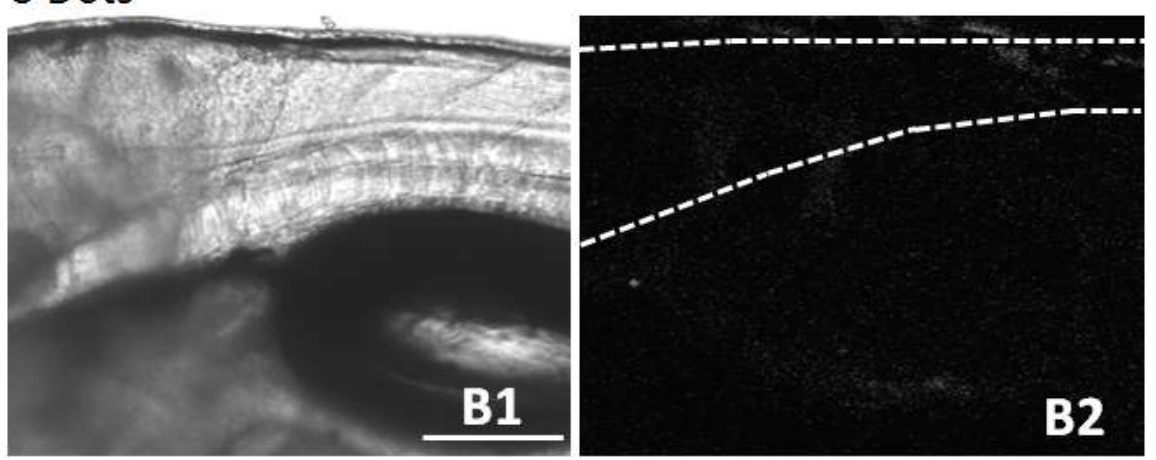

\section{Dye-transferrin-C-Dots}
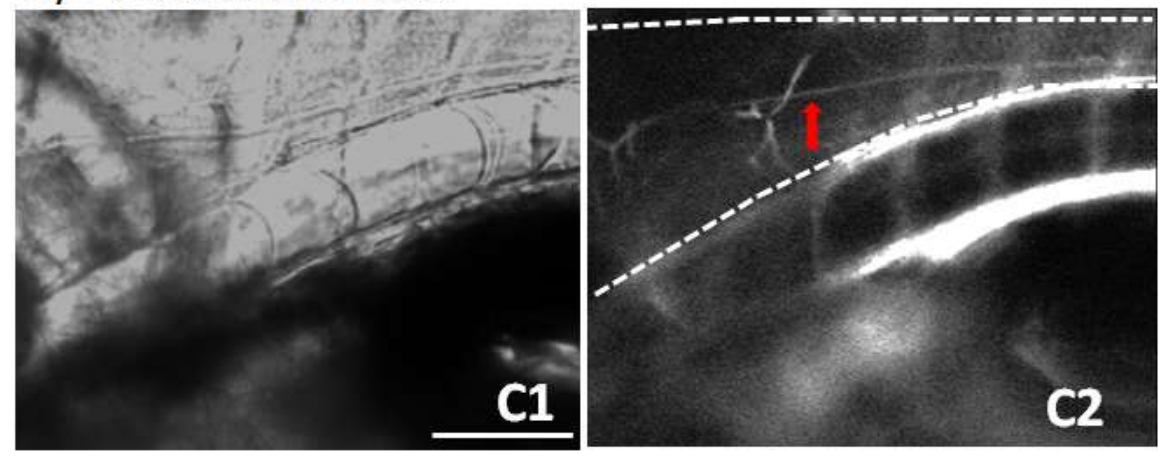
Figure 3. Confocal microscopic images of zebrafish under visible light (A1, B1 and C1), excitation of C-Dots at $458 \mathrm{~nm}(\mathrm{~A} 2$ and B2), and excitation of the dye at $561 \mathrm{~nm}(\mathrm{C} 2)$. A1 and A2: zebrafish without injection; B1 and B2 with injection of C-Dots; and C1 and C2 with injection of dye-transferrin-C-Dots. The dashed line shows the CNS zone of zebrafish. The red arrow in Figure $3 \mathrm{C} 2$ points to the central canal. In each figure, the scale bar is $100 \mu \mathrm{m}$.

Receptor-mediated endocytosis has previously been utilized for efficient drug delivery to the target cells with high expression of the receptors. ${ }^{40,41}$ As an example, transferrin receptor has before been explored as a target to deliver therapeutics into the cancer cells and brains. ${ }^{41}$ It is reported that transferrin receptor is over-expressed on BBB. ${ }^{42}$ In addition, the expression of transferrin receptor on the BBB in the larval zebrafish is active at 6 days old. Therefore in this study C-Dots were covalently conjugated to transferrin with the goal of allowing C-Dots to cross the $\mathrm{BBB}$ via transferrin receptor-mediated endocytosis. To image the presence of these materials in the CNS we focused on the plane of the notochord, a large structure in the middle of the fish that provides structural support for the CNS. When we injected the transferrin-conjugated C-Dots under the same conditions as the injection of C-Dots only, no fluorescence difference was observed (Figure SI-3 E2). Even if the conjugates could enter the CNS, no obvious fluorescence would be observed due to the weak fluorescence of C-Dots. Therefore, transferrin labeled with a fluorescent dye was selected to better illustrate the possible entry of the CNS. Following the same procedures, the dye labeled conjugation system was injected to the heart of zebrafish. In this case, fluorescence could be seen in the CNS, with the brightest signal in the central canal (Figure $3 \mathrm{C} 2$ ). In addition to the central canal, faint fluorescence can be seen surrounding neuronal cell bodies. The central canal of the spinal cord was quite clear (red arrows in Figure $3 \mathrm{C} 2$ ). This was not observed in Figure 3 B2 and Figure SI-3 D2 and E2. Therefore, the conjugation system of dyetransferrin-C-Dots was delivered to the CNS. To further support this conclusion, the dyetransferrin-C-Dots were injected to the zebrafish heart which had green fluorescent protein (GFP) labeled neurons. Under the excitation of GFP and the red dye, one could observe simultaneously the green fluorescence of neurons and the red dye fluorescence (Figure 4). The white arrow showed the central canal, which was surrounded by the green fluorescent neuron cells in the CNS zone (the white arrow shown in Figure 4). These experimental results indicated that the conjugates could enter the CNS by crossing the BBB. 

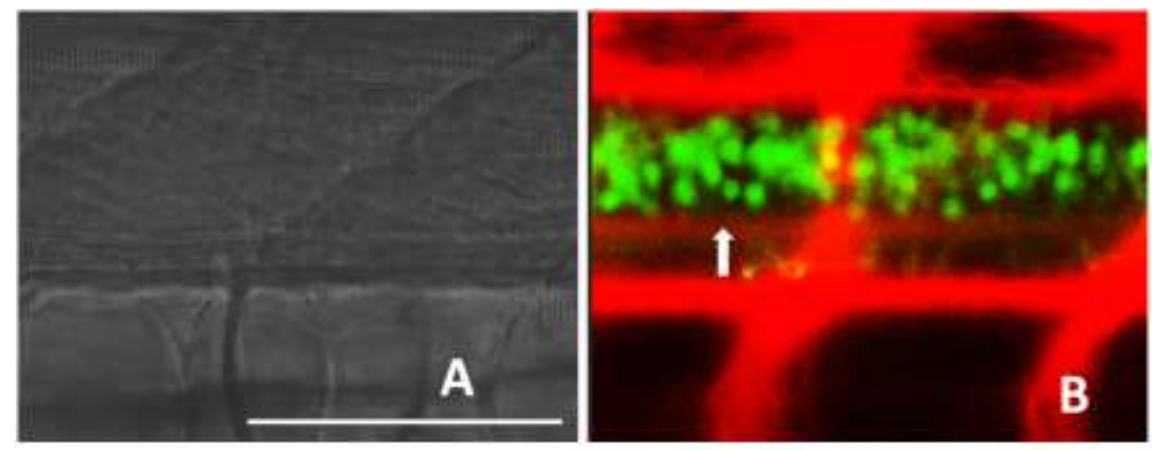

Figure 4. Confocal microscopic images of zebrafish under visible light (A), excitation of GFP expressed in a subset of CNS interneurons at $488 \mathrm{~nm}$ (B, green), and excitation of the dye at 561 $\mathrm{nm}(\mathrm{B}, \mathrm{red})$. The arrow in B points to the central canal. In both figures, the scale bar is $100 \mu \mathrm{m}$.

Since C-Dots are able to suppress the process of protein fibrillation, ${ }^{22}$ the conjugation of transferrin-C-Dots system can be potentially used in future research to enter the CNS and prevent the fibrillation of the neurodegenerative proteins. The fibrillation of some proteins (e.g. $\alpha$ synuclein and amyloid beta 40/42) is known to be associated with the pathology of human neurodegenerative disorders, such as Parkinson's and Alzheimer's diseases. ${ }^{43}$ Since remarkable anatomical and physiological conservation has been documented in CNS development and function between zebrafish and mammals (e.g. mouse and humans), this study of zebrafish model indicates that transferrin-C-Dots system could be delivered to the CNS of mammals via a similar mechanism.

C-Dots have shown potential for drug delivery and treatment in some CNS related diseases. Nevertheless, the major challenge for these applications is delivery of C-Dots to the CNS. Crossing the blood-brain barrier (BBB) is inhibited and therefore therapeutic agents may be blocked before reaching the pathological tissues in the CNS. Previous to this study, the question as to whether C-Dots or C-Dots conjugates can cross the BBB to enter the CNS had not been examined. In this study, C-Dots were covalently conjugated to transferrin as well as dye labeled transferrin to examine the potential of crossing the $\mathrm{BBB}$ via the transferrin receptor-mediated delivery in the zebrafish model. The experimental observations from this in vivo study suggest that the transferrin conjugated C-Dots can enter the CNS by crossing the BBB but C-Dots alone cannot. This study provides a strategy by which delivery of C-Dots across the BBB to the CNS can be achieved.

\section{Acknowledgements}


R.M.L. gratefully acknowledges the support of the National Science Foundation under Grant 1355317.

Supporting Information including experimental materials and methods is available online.

\section{Reference}

(1) Hawkins, B. T.; Davis, T. P. Pharmacol. Rev. 2005, 57, 173.

(2) Brightman, M. W.; Reese, T. S. J. Cell Biol. 1969, 40, 648.

(3) Pardridge, W. M. Drug Discov. Today 2002, 7, 5.

(4) Pardridge, W. M. NeuroRx 2005, 2, 3.

(5) Wohlfart, S.; Gelperina, S.; Kreuter, J. J. Control. Release 2012, 161, 264.

(6) Huang, H. Y.; Liu, H. L.; Hsu, P. H.; Chiang, C. S.; Tsai, C. H.; Chi, H. S.; Chen, S. Y.; Chen, Y. Y. Adv. Mater. 2015, 27, 655.

(7) Li, J. Y.; Sugimura, K.; J.Boado, R.; Lee, H. J.; Zhang, C.; Duebel, S.; M.Pardridge, W. Protein Eng. 1999, 12, 787.

(8) Cao, C.; Wang, X.; Cai, Y.; Sun, L.; Tian, L.; Wu, H.; He, X.; Lei, H.; Liu, W.; Chen, G.; Zhu, R.; Pan, Y. Adv. Mater. 2014, 26, 2566.

(9) Kreuter, J. Adv. Drug Deliv. Rev. 2014, 71, 2.

(10) Rubin, L.; Staddon, J. Annu. Rev. Neurosci. 1999, 22, 11.

(11) Wiley, D. T.; Webster, P.; Gale, A.; Davis, M. E. Proc. Natl. Acad. Sci. U.S.A. 2013, 110, 8662.

(12) Roberts, R. L.; Fine, R. E.; Sandra, A. J Cell Sci. 1993, 104, 521.

(13) Yu, Y. J.; Watts, R. J. Neurotherapeutics 2013, 10, 459.

(14) Salvati, A.; Pitek, A. S.; Monopoli, M. P.; Prapainop, K.; Bombelli, F. B.; Hristov, D. R.; Kelly, P. M.; Aberg, C.; Mahon, E.; Dawson, K. A. Nat. Nano. 2013, 8, 137.

(15) Dixit, S.; Novak, T.; Miller, K.; Zhu, Y.; Kenney, M. E.; Broome, A.-M. Nanoscale 2015, 7, 1782 .

(16) Jiang, W.; Xie, H.; Ghoorah, D.; Shang, Y.; Shi, H.; Liu, F.; Yang, X.; Xu, H. PLoS ONE 2012, 7, e37376.

(17) Gu, F. X.; Karnik, R.; Wang, A. Z.; Alexis, F.; Levy-Nissenbaum, E.; Hong, S.; Langer, R. S.; Farokhzad, O. C. Nano Today 2007, 2, 14.

(18) Schwenke, A. M.; Hoeppener, S.; Schubert, U. S. Adv. Mater. 2015, 27, 4113.

(19) Xu, X.; Ray, R.; Gu, Y.; Ploehn, H. J.; Gearheart, L.; Scrivens, W. A. J. Am. Chem. Soc 2004, 126, 12736. 
(20) Yang, S.-T.; Wang, X.; Wang, H.; Lu, F.; Luo, P. G.; Cao, L.; Meziani, M. J.; Liu, J.-H.; Liu, Y.; Chen, M.; Huang, Y.; Sun, Y.-P. J. Phys. Chem. C 2009, 113, 18110.

(21) Li, Q.; Ohulchanskyy, T. Y.; Liu, R.; Koynov, K.; Wu, D.; Best, A.; Kumar, R.; Bonoiu, A.; Prasad, P. N. J. Phys. Chem. C 2010, 114, 12062.

(22) Li, S.; Wang, L.; Chusuei, C. C.; Suarez, V. M.; Blackwelder, P. L.; Micic, M.; Orbulescu, J.; Leblanc, R. M. Chem. Mater. 2015, 27, 1764.

(23) Chiti, F.; Dobson, C. M. Annu. Rev. Biochem. 2006, 75, 333.

(24) Li, S.; Leblanc, R. M. J. Phys. Chem. B 2014, 118, 1181.

(25) Zheng, M.; Ruan, S.; Liu, S.; Sun, T.; Qu, D.; Zhao, H.; Xie, Z.; Gao, H.; Jing, X.; Sun, Z. ACS Nano 2015, 9, 11455.

(26) Panula, P.; Sallinen, V.; Sundvik, M.; Kolehmainen, J.; Torkko, V.; Tiittula, A.; Moshnyakov, M.; Podlasz, P. Zebrafish 2006, 3, 235.

(27) Gribble, S. L.; Nikolaus, O. B.; Dorsky, R. I. Dev. Dyn. 2007, 236, 3472.

(28) Santoriello, C.; Zon, L. I. J. Clin. Invest. 2012, 122, 2337.

(29) Aceto, J.; Nourizadeh-Lillabadi, R.; Marée, R.; Dardenne, N.; Jeanray, N.; Wehenkel, L.; Aleström, P.; van Loon, J. J. W. A.; Muller, M. PLoS ONE 2015, 10, e0126928.

(30) Xie, J.; Farage, E.; Sugimoto, M.; Anand-Apte, B. BMC Dev. Biol. 2010, 10, 76.

(31) Jeong, J.-Y.; Kwon, H.-B.; Ahn, J.-C.; Kang, D.; Kwon, S.-H.; Park, J. A.; Kim, K.-W. Brain Res. Bull. 2008, 75, 619.

(32) Li, S.; Peng, Z.; Leblanc, R. M. Anal. Chem. 2015, 87, 6455.

(33) James, N. G.; Berger, C. L.; Byrne, S. L.; Smith, V. C.; MacGillivray, R. T. A.; Mason, A. B. Biochemistry 2007, 46, 10603.

(34) Yang, Z.-C.; Wang, M.; Yong, A. M.; Wong, S. Y.; Zhang, X.-H.; Tan, H.; Chang, A. Y.; Li, X.; Wang, J. Chem. Commun. 2011, 47, 11615.

(35) Nie, H.; Li, M.; Li, Q.; Liang, S.; Tan, Y.; Sheng, L.; Shi, W.; Zhang, S. X.-A. Chem. Mater. 2014, 26, 3104.

(36) Zhu, S.; Meng, Q.; Wang, L.; Zhang, J.; Song, Y.; Jin, H.; Zhang, K.; Sun, H.; Wang, H.; Yang, B. Angew. Chem. Int. Ed. 2013, 52, 3953.

(37) Aubin-Tam, M.-E.; Hwang, W.; Hamad-Schifferli, K. Proc. Natl. Acad. Sci. U.S.A. 2009, 106,4095 .

(38) Abbott, N. J.; Ronnback, L.; Hansson, E. Nat. Rev. Neurosci. 2006, 7, 41.

(39) Chang, J. T.; Lehtinen, M. K.; Sive, H. Dev. Neurobiol. 2016, 76, 75.

(40) Daniels, T. R.; Delgado, T.; Helguera, G.; Penichet, M. L. Clin. Immunol. 2006, 121, 159.

(41) Qian, Z. M.; Li, H.; Sun, H.; Ho, K. Pharmacol. Rev. 2002, 54, 561. 
(42) Moos, T.; Morgan, E. H. Cell Mol. Neurobiol. 2000, 20, 77.

(43) Irvine, G. B.; El-Agnaf, O. M.; Shankar, G. M.; Walsh, D. M. Mol. Med. 2008, 14, 451. 